Aim of the study: To evaluate the toxicity, clinical effectiveness and survival rate of transurethral resection, neoadjuvant chemotherapy and accelerated hyperfractionated radiotherapy (concomitant boost), with or without concurrent cisplatin in patients with muscle invasive bladder cancer.

Material and methods: Between March 2004 and December 2009, 35 patients with histologically proven invasive carcinoma of the bladder (T2-4a, NO-1, MO), who were fit for combined radiochemotherapy and refused radical surgery or were medically or surgically inoperable, were selected for the bladder-sparing protocol. Results: In this study, twenty-five patients (25/35; $72 \%)$ received two cycles of neoadjuvant chemotherapy, and ten of them $(10 / 35 ; 28 \%)$ only one, because of treatment-related toxicity. In twenty-one patients $(21 / 35 ; 60 \%)$ chemotherapy consisting of gemcitabine with cisplatin and in fourteen patients $(14 / 35$; $40 \%)$ gemcitabine with carboplatin were applied. Only 13 patients $(13 / 35 ; 37 \%)$ received combined irradiation with cisplatin. All patients completed their planned course of radiation therapy. Complete response (CR) occurred in $26 / 35$ (74\%) patients, partial response (PR) in $2 / 35(6 \%)$, and stable disease (SD) in 7/35 (20\%). The overall actuarial survival rates at 3 and 5 years were $75 \%$ and $66 \%$, respectively. Disease-specific actuarial survival rates at 3 and 5 years were $81 \%$ and $71 \%$, respectively.

Conclusions: Conservative treatment of patients with muscle-invasive bladder cancer by transurethral resection, neoadjuvant chemotherapy, and accelerated hyperfractionated radiotherapy with concomitant boost, with or without concurrent cisplatin, provides a high probability of local and distal response with acceptable toxicity in properly selected patients.

Key words: bladder cancer, transurethral resection, chemotherapy, radiotherapy.

\section{Transurethral resection, neoadjuvant chemotherapy and accelerated hyperfractionated radiotherapy (concomitant boost), with or without concurrent cisplatin, for patients with invasive bladder cancer - clinical outcome}

\author{
Jadwiga Nowak-Sadzikowska, Jerzy Jakubowicz, Tomasz Skóra, \\ Katarzyna Pudełek
}

Centre of Oncology - Maria Skłodowska-Curie Memorial Institute, Krakow Branch, Krakow, Poland

\section{Introduction}

Radical cystectomy remains the gold standard treatment for invasive bladder cancer, despite encouraging results of bladder preservation regimens in a series of single institution and cooperative group trials [1-6]. However, no randomized trial has ever been conducted to directly compare cystectomy and selective bladder-sparing therapy. It should be pointed out that patients selected for a conservative approach have worse performance status, are relatively older, with more advanced clinical stage and significant comorbidities, compared with those treated by surgery.

Combined modality bladder-sparing treatment can achieve complete response rates of $60-85 \%$ and 5 -year overall survival rates of $50-63 \%$, and approximately $75 \%$ of surviving patients maintained their bladder [1-12]. The most optimal bladder-preserving approach combines maximal transurethral resection of bladder cancer (TURB), followed by radiation therapy (RT) and systemic chemotherapy (CT), with cystectomy reserved for salvage. For patients who are not surgical candidates or refused surgery, primary local treatment alone (TURB plus radiation therapy) has afforded clinical complete response rates of $40-50 \%$ and 5 -year survival rates of $20-40 \%$. Many studies have been performed to improve the results of radiotherapy in treatment of invasive bladder cancer by escalation of total dose, adoption of altered fractionation, reduction in overall treatment time, combining teleradiotherapy with brachytherapy or using radiosensitizers. In attempts to obtain better results of local treatment of invasive bladder cancer systemic chemotherapy has been incorporated due to low local control and a high rate of distant metastases. The optimal management of chemotherapy and radiation therapy delivery, sequentially or concurrently, remains to be determined.

We designed a bladder-sparing treatment protocol consisting of transurethral resection, neoadjuvant chemotherapy with gemcitabine and cisplatin or carboplatin, and accelerated hyperfractionated radiotherapy (concomitant boost) with concurrent cisplatin, in an effort to improve both local and distant control.

\section{Material and methods}

Between March 2004 and December 2009, 35 patients with histologically confirmed invasive bladder cancer (T2-4a,NO-1,M0), who were fit for com- 
bined radiochemotherapy and refused radical surgery or were medically or surgically inoperable, were selected for bladdersparing treatment. Exclusion criteria were as follows: Karnofsky status $<70 \%$, haemoglobin $<10 \mathrm{~g} / \mathrm{dl}$, white blood cell count $<4000 / \mathrm{ml}$, platelet count $<100,000 / \mathrm{ml}$, serum bilirubin and serum creatinine level above the upper limit of normal, previous radiotherapy to the pelvis. Prior to therapy, all patients underwent physical examination with DRE (digital rectal examination), cystoscopy with TURB, haematological, renal and biochemical blood tests, CT scan of abdomen and pelvis, and chest X-ray.

The pre treatment patient and tumour characteristics were as follows: mean age 63 years (range 46-76); 29/35 (83\%) male, $6 / 35$ (17\%) female; Karnofsky status 70 - 18/35 (51\%), $80-13 / 35$ (37\%), $\geq 90-4 / 35(12 \%)$, clinical stage $T_{2}$ - 8/35 (23\%), $\mathrm{T}_{3}-17 / 35$ (49\%), $\mathrm{T}_{4}-10 / 35$ (28\%); $7 / 35$ (20\%) with hydronephrosis and 28/35 (80\%) without.

Patients underwent standard transurethral resection of bladder tumour (TURB), and a complete TURB was attempted, if possible. Visibly complete TURB was achieved in $12 / 35$ (34\%). The histological grade findings were $G_{2} 11 / 35$ (31\%) and $\mathrm{G}_{3} 24 / 35$ (69\%).

Neoadjuvant chemotherapy, two cycles with gemcitabine and cisplatin, was started within 1-8 weeks (median 2 weeks) after maximal TURB. Patients received gemcitabine $1000 \mathrm{mg} / \mathrm{m}^{2}$ over 30 to 60 minutes on days 1,8 , and 15, plus cisplatin $70 \mathrm{mg} / \mathrm{m}^{2}$ on day 2. Cycles were repeated every 28 days.

In patients with abnormal renal function and/or severe heart disease chemotherapy with gemcitabine and carboplatin was used. Gemcitabine $1000 \mathrm{mg} / \mathrm{m} 2$ over 30 to $60 \mathrm{~min}$ utes on days 1,8 , and 15, plus carboplatin dosed at an area under the curve (AUC) of 5 over 60 minutes on day 2 were given. Cycles were repeated every 28 days. Twenty-one patients (60\%) in this group received chemotherapy consisting of gemcitabine with cisplatin and fourteen patients (40\%) gemcitabine with carboplatin.

External beam RT with computed tomography-based images was performed with $6 \mathrm{MV}$ or $18 \mathrm{MV}$ photon beams from the linear accelerator. Radiotherapy was started about 4-6 weeks after neoadjuvant chemotherapy. The irradiation dose per fraction was 1.8 Gy daily, to a total of 45 Gy to the whole bladder with $2-3 \mathrm{~cm}$ margin, for 5 weeks $-25 \times 1.8 \mathrm{~Gy}$, once daily, 5 days a week. Additionally, all patients received a concomitant boost to the whole bladder with 1-1.5 cm margin, during the two last weeks of treatment, as a second fraction to the total dose of $60 \mathrm{~Gy}$, and a 6-hour interval between two fractions a day was mandatory. A four-field or a threefield technique was used with individually shaped portals by multi-leaf collimators (MLC). Patients were treated with an empty bladder.

Concurrent chemotherapy was applied on days 1, 2, 15, $16,29,30$ of radiotherapy and consisted of cisplatin $20 \mathrm{mg} / \mathrm{m}^{2}$ in $30 \mathrm{~min}$ infusion, 3-4 hours before irradiation. From 2008 , cisplatin $20 \mathrm{mg} / \mathrm{m}^{2}$ in 30 min infusion on days $1,7,14$, $21,28,35$ of radiotherapy was administered. Only 13 patients $13 / 35$ (37\%) received combined irradiation with cisplatin. The remaining patients were not treated with cisplatin during irradiation, due to exacerbation of chronic comorbid diseases.

The treatment scheme in a group of 35 patients with invasive bladder cancer is presented in Table 1 .
Table 1. Treatment scheme in a group of 35 patients with invasive bladder cancer

\begin{tabular}{lc}
\hline Treatment scheme & $\begin{array}{c}\text { No. of } \\
\text { patients (\%) }\end{array}$ \\
$\begin{array}{lc}\text { TURB + neoadjuvant chemotherapy with } \\
\text { gemcitabine and cisplatin + combined irradiation } \\
\text { with cisplatin }\end{array}$ & $9(26)$ \\
\hline $\begin{array}{l}\text { TURB + neoadjuvant chemotherapy with gemcitabine } \\
\text { and cisplatin + irradiation alone }\end{array}$ & $12(34)$ \\
\hline $\begin{array}{l}\text { TURB + neoadjuvant chemotherapy with gemcitabine } \\
\text { and carboplatin + combined irradiation with cisplatin }\end{array}$ & $4(11)$ \\
\hline $\begin{array}{l}\text { TURB + neoadjuvant chemotherapy with gemcitabine } \\
\text { and carboplatin + irradiation alone }\end{array}$ & $10(29)$ \\
\hline total & $35(100)$
\end{tabular}

Acute toxicities were recorded every week according to the Common Toxicity Criteria for Adverse Events [13].

Initial treatment response was assessed 4-6 weeks after neoadjuvant chemotherapy, by re-evaluation of tumour size by computed tomography of pelvis.

Six to eight weeks after completion of the concervative treatment, response was evaluated by control cystoscopy with biopsy of the former tumour region or TURB. In case of a histologically proven complete response, patients were followed up at 3-month intervals for the first 3 years and every 6 months for 5 years and then annually. Evaluations consisted of physical examination and cystoscopy, with biopsies only if clinically indicated. Each year, abdominopelvic computed tomography, chest X-ray or other instrumental examinations, if indicated, were performed.

For persistent or recurrent invasive tumour, salvage cystectomy was recommended, while for superficial recurrences, TURB and intravesical therapy were advised.

Actuarial survival probabilities were calculated using the Kaplan-Meier method [14]. Overall survival (OS) was calculated from the date of treatment initiation to the date of death of any cause, or censor date for alive patients. Disease-specific survival (DSS) was defined as the duration from the date of treatment initiation to the date of death from bladder cancer or last follow-up. Bladder-intact survival (BIS) was defined as the interval between treatment initiation and cystectomy or death or last follow-up if no event occurred.

\section{Results}

\section{Acute toxicity of treatment}

Twenty-five patients (25/35; $72 \%$ ) received two cycles of neoadjuvant chemotherapy, and ten of them (10/35; 28\%) only one, because of treatment-related toxicity. During neoadjuvant chemotherapy typical acute haematological toxicity, mainly grade 1-2, was presented. Grade 1-2 haematological side effects included anaemia in 6/35 (17\%), leucopenia in 20/35 (56\%), neutropenia in 9/35 (26\%) and thrombocytopenia in $6 / 35$ (17\%) patients. Grade 3 toxicities in terms of leucopenia occurred in 1/35 (3\%), neutropenia in $8 / 35$ (23\%) and thrombocytopenia in $8 / 35$ (23\%) patients. In one patient, neoadjuvant chemotherapy was discontinued after the first cycle, due to severe acute toxicity - leucopenia $G_{4}$, 
Table 2. Response rate and failure pattern related to treatment scheme in a group of 35 patients with invasive bladder cancer

\begin{tabular}{|c|c|c|c|c|}
\hline \multirow{2}{*}{$\begin{array}{l}\text { Treatment scheme } \\
\text { patient No. (\%) }\end{array}$} & \multirow{2}{*}{$\begin{array}{c}\text { Response } \\
\text { patient No. (\%) }\end{array}$} & \multicolumn{3}{|c|}{ Failure patient No. } \\
\hline & & $\begin{array}{l}\text { Invasive bladder } \\
\text { recurrence }\end{array}$ & $\begin{array}{l}\text { Superficial } \\
\text { relapse }\end{array}$ & Dissemination \\
\hline $\begin{array}{l}\text { TURB + neoadjuvant chemotherapy + } \\
\text { combined irradiation with cisplatin } \\
13 \text { (37) }\end{array}$ & $\begin{array}{l}\text { CR } 7(20) \\
\text { PR } 1(3) \\
\text { SD } 5(14)\end{array}$ & 1 & 3 & $\begin{array}{l}2 \\
- \\
4\end{array}$ \\
\hline $\begin{array}{l}\text { TURB + neoadjuvant chemotherapy + } \\
\text { irradiation alone } \\
22(63)\end{array}$ & $\begin{array}{l}\text { CR } 19(54) \\
\text { PR } 1(3) \\
\text { SD } 2(6)\end{array}$ & 2 & 3 & $\begin{array}{l}1 \\
1 \\
1\end{array}$ \\
\hline $\begin{array}{l}\text { total } \\
35(100)\end{array}$ & $\begin{array}{l}\text { CR } 26(74) \\
\text { PR } 2(6) \\
\text { SD } 7(20)\end{array}$ & 3 & 6 & $\begin{array}{l}3 \\
1 \\
5\end{array}$ \\
\hline
\end{tabular}

neutropenia $\mathrm{G}_{4}$, gastrointestinal toxicity $\mathrm{G}_{4}$. Modification of doses, or delay or omission in administration of cytostatics was reported in 27 patients 27/35 (77\%). Granulocytic colony-stimulating factors were used in 11 patients $(11 / 35 ; 31 \%)$ to preserve the regime of chemotherapy.

All patients completed their planned course of radiation therapy. There were no complications worse than grade 2 in this group, during irradiation or chemoradiation, except one G3 genitourinary toxicity. 19/35 (54\%) patients developed grade $1-2$ gastrointestinal and $28(80 \%)$ genitourinary.

\section{Response to therapy and failure pattern}

Initial treatment response, assessed after neoadjuvant chemotherapy, presented complete response in 8 patients (8/35; 23\%), partial response in 19/35 (54\%), and stable disease in $8 / 35$ (23\%).

Complete response (CR) after finished conservative treatment - transurethral resection, neoadjuvant chemotherapy with gemcitabine and cisplatin or carboplatin, and accelerated hyperfractionated radiotherapy with concomitant boost with or without concurrent cisplatin - occurred in 26/35 (74\%) patients, partial response (PR) in 2/35 (6\%), and stable disease (SD) in 7/35 (20\%). The group without complete response consisted of T3-T4 patients, except one T2. Response rate and failure pattern related to treatment scheme are shown in Table 2.

In a group of 9 patients with persistent disease, two were salvaged with cystectomy. The rest of them were unfit for surgery due to stage of disease and/or performance status. They were treated by transurethral resection and/or systemic chemotherapy.

Three of 26 patients who were clinical complete responders had invasive bladder recurrence. Superficial relapse occurred in 6 patients. Median time to local failure was 26 months (range 15 to 77 months). Two patients with invasive recurrence underwent salvage cystectomy; one was treated by transurethral resection. Patients with superficial relapse were treated by transurethral resection followed or not followed by endovesical therapy.

Metastases developed in 9 patients, without associated local failure in 2 cases.

\section{Survival}

At the time of analysis median follow-up for surviving patients was 46 months. There were 10 deaths in the whole group.

The overall actuarial survival rates at 3 and 5 years were $75 \%$ and $66 \%$, respectively. Disease-specific actuarial survival rates at 3 and 5 years were $81 \%$ and $71 \%$, respectively. Bladder-intact survival at 3 and 5 years were $72 \%$ and $63 \%$, respectively.

\section{Discussion}

In the current series, the 5 -year overall survival rate of $66 \%$ and disease-specific survival rate of $71 \%$ are similar to the results of others [1-12].

We observed a substantial percentage of response failure, and finally poor results in patients with locally advanced tumour T3-T4. Of the patients with incomplete response $(9 / 35 ; 26 \%)$, only two were treated with salvage cystectomy. The rest of them were considered unfit for radical cystectomy due to stage of disease and/or medical contraindications or refused surgery. For patients with tumour persistence or with invasive recurrence, close cystoscopic surveillance with prompt salvage cystectomy is an essential part of the bladder preservation strategy which allows to obtain long-term results. In our series only four patients (4/35; 11\%) were treated with salvage cystectomy due to incomplete response or local failure, which is a considerably low number compared with other series. In published studies salvage cystectomy was done when necessary in about 20-35\% of all patients treated by selective bladder preservation $[1,4,5]$. The decision to remove the bladder should not be deferred if no complete response is achieved. The second curative approach by salvage surgery in case of local failure can be performed safely, with comparable risk of complications to primary cystectomy.

Close cooperation between urologic surgeons, radiation oncologists and medical oncologists seems to be most important in order to succeed in organ preservation therapy for muscle invasive bladder cancer.

Our results are also comparable to contemporary radical cystectomy series [15]. It should be noted however, that direct comparison to cystectomy is confounded by the discordance 
between clinical and pathologic staging [16, 17]. Despite the innovation of modern methods of imaging, TURB constitutes the most important part of clinical staging. However, all parts of clinical staging are connected with a high percentage of errors. The clinical staging is more likely to understage the extent of disease with regard to penetration into muscular propria or bladder surrounding fat.

Despite the many randomized controlled trials carried out, controversy still exists as to whether neoadjuvant chemotherapy improves survival in patients with invasive bladder cancer treated conservatively [6, 7-9]. The international phase III trial assessing neoadjuvant cisplatin, methotrexate, and vinblastine chemotherapy (CMV) for muscle-invasive bladder cancer BA06 30894 showed a statistically significant 16\% reduction in the risk of death ( $\mathrm{HR} 0.84 ; \mathrm{Cl}$ : 0.72-0.99; $p=0.037$ ), corresponding to an increase in 10-year survival from $30 \%$ to $36 \%$ [6]. A meta-analysis assessing the effect of neoadjuvant combination, platinum-based chemotherapy in treatment of invasive bladder cancer showed a significant benefit for overall survival, which increased from $45 \%$ to $50 \%$, and reduced risk of death by $13 \%$ [12]. This effect was observed irrespective of the type of local treatment. However, the results of a randomized trial (RTOG 89-03) comparing two treatment arms of RT and concurrent cisplatin with or without neoadjuvant chemotherapy, using the CMV scheme, reported no differences in terms of local control, survival without metastases and overall survival [13]. The presented data suggested that prolongation of treatment time could have an unfavourable effect.

Clinical research on using cisplatin in combination with $\mathrm{RT}$, in patients with invasive bladder cancer, has demonstrated objective tumour responses of up to $80 \%$, with acceptable toxicity [2, 5, 10-11]. In a prospective study, use of a daily low dose of cisplatin $\left(6 \mathrm{mg} / \mathrm{m}^{2}\right)$ with accelerated radiotherapy, in patients with invasive bladder cancer, was feasible with acceptable tolerance, even in relatively old patients [11].

With only 13 patients $(13 / 35 ; 37 \%)$ who received combined chemoradiotherapy with cisplatin after neoadjuvant chemotherapy consisting of gemcitabine with cisplatin or gemcitabine with carboplatin, this regimen appears to be poorly tolerated by most patients. The toxicity of cisplatin significantly limits its utility. The rate of chemoradiotherapy is also adversely impacted by toxicity of neoadjuvant chemotherapy.

Many studies have been conducted to improve the results of radiotherapy in treatment of invasive bladder cancer by adoption of altered fractionation, to reduce overall treatment time and acceleration of clonogenic repopulation of tumour [19-23]. One of the methods of altered fractionation, used in treatment of invasive bladder cancer, is accelerated superfractionated radiotherapy with concomitant boost, which allows to obtain a complete response in about $74-80 \%$ of invasive bladder carcinoma patients, with acceptable toxicity $[19,20]$.

On the other hand, some authors consider that the effect of the overall treatment time (OTT) in invasive bladder cancer is difficult to define [21] or they even do not find a statistically significant correlation between local control and treatment time [22]. The results of a published randomised phase III trial comparing accelerated with conventional fractionation, showed no improvement of the accelerated arm [23].
Another strategy is to target tumour cell repopulation by the addition of chemotherapy to radiotherapy [1-6, 10-12]. Rapid proliferation is associated with improved local control, if patients are treated with concurrent radiochemotherapy [24]. The cytotoxic effect of concurrent chemotherapy may effectively inhibit repopulation during fractionated radiotherapy. To enhance this effect, chemotherapy with accelerated radiotherapy protocols were designed, in an effort to improve both local and distant control [25-26].

In the trial RTOG 97-06, in which patients were treated with twice daily accelerated irradiation with cisplatin, and adjuvant CMV (methotrexate, vinblastine and cisplatin) and trial RTOG 99-06, using twice daily radiotherapy with cisplatin and paclitaxel and adjuvant cisplatin and gemcitabine, the complete response rate achieved ranged from 74 to $81 \%[25,26]$.

In our series, a CR rate of $74 \%$ was observed, which is similar to that in other bladder sparing regimens. However, it should be noted that the majority of treated patients were in an advanced stage of disease $-77 \%$ of treated patients with T3-T4.

Acute bowel and urinary toxicity during accelerated hyperfractionated radiotherapy with concomitant boost for invasive bladder cancer were observed in 74-95\% and 74-91\% of the patients, respectively, which is consistent with our observations $[19,20]$. We observed acute bowel and urinary toxicity, mostly grade 2 , in $54 \%$ and $80 \%$, respectively, except one $\mathrm{G} 3$ genitourinary. All patients received planned irradiation with the prescribed dose, at the schedule time.

In conclusion, conservative treatment of patients with muscle-invasive bladder cancer by transurethral resection, neoadjuvant chemotherapy, and accelerated hyperfractionated radiotherapy with concomitant boost, with or without concurrent cisplatin, provides a high probability of local and distal response with acceptable toxicity in properly selected patients who are unwilling or unfit to undergo radical cystectomy. Close surveillance after multimodality bladder preservation therapy with salvage cystectomy for nonresponders or recurrence is an integral and important part of this treatment.

The authors declare no conflict of interest.

\section{References}

1. Rödel C, Grabenbauer GG, Kühn R, Papadopoulos T, Dunst J, Meyer M, Schrott KM, Sauer R. Combined-modality treatment and selective organ preservation in invasive bladder cancer: long-term results. J Clin Oncol 2002; 20: 3061-71.

2. Perdoní S, Autorino R, Damiano R, et al. Bladder-sparing, combinedmodality approach for muscle-invasive bladder cancer: a multi-institutional, long-term experience. Cancer 2008; 112: 75-83.

3. James ND, Hussain SA, Hall E, et al. Radiotherapy with or without chemotherapy in muscle-invasive bladder cancer. N EngI J Med 2012; 366: 1477-88.

4. Efstathiou JA, Spiegel DY, Shipley WU, et al. Long-term outcomes for selective bladder preservation by combined-modality therapy for invasive bladder cancer: the MGH experience. Eur Urol 2012; 61: 705-11.

5. Shipley WU, Kaufman DS, Zehr E, Heney NM, Lane SC, Thakral HK, Althausen AF, Zietman AL. Selective bladder preservation by combined modality protocol treatment: long-term outcomes of 190 patients with invasive bladder cancer. Urology 2002; 60: 62-7. 
6. International Collaboration of Trialists; Medical Research Council Advanced Bladder Cancer Working Party (now the National Cancer Research Institute Bladder Cancer Clinical Studies Group); European Organisation for Research and Treatment of Cancer Genito-Urinary Tract Cancer Group; Australian Bladder Cancer Study Group; National Cancer Institute of Canada Clinical Trials Group; Finnbladder; Norwegian Bladder Cancer Study Group; Club Urologico Espanol de Tratamiento Oncologico Group, Griffiths G, Hall R, Sylvester R, Raghavan D, Parmar MK. International phase III trial assessing neoadjuvant cisplatin, methotrexate, and vinblastine chemotherapy for muscle-invasive bladder cancer: long-term results of the BA06 30894 trial. J Clin Oncol 2011; 16: 2171-7.

7. Sherif A, Rintala E, Mestad O, Nilsson J, Holmberg L, Nilsson S, Malm ström PU; Nordic Urothelial Cancer Group. Neoadjuvant cisplatinmethotrexate chemotherapy for invasive bladder cancer - Nordic cystectomy trial 2. Scand J Urol Nephrol 2002; 6: 419-25.

8. Advanced Bladder Cancer Meta-analysis Collaboration. Neoadjuvant chemotherapy in invasive bladder cancer: a systematic review and meta-analysis. Lancet 2003; 361: 1927-34

9. Shipley WU, Winter KA, Kaufman DS, et al. Phase III trial of neoadjuvant chemotherapy in patients with invasive bladder cancer treated with selective bladder preservation by combined radiation therapy and chemotherapy: initial results of Radiation Therapy Oncology Group 89-03. 89-03. J Clin Oncol 1998; 16: 3576-83.

10. Coppin CM, Gospodarowicz MK, James K, Tannock IF, Zee B, Carson J, Pater J, Sullivan LD. Improved local control of invasive bladder cancer by concurrent cisplatin and preoperative or definitive radiation: The National Cancer Institute of Canada Clinical Trials Group. J Clin Oncol 1996; 14: 2901-07.

11. Zouhair A, Ozsahin M, Schneider D, Bauer J, Jichlinski P, Roth A, Dou glas P, Miralbell R. Invasive bladder carcinoma: a pilot study of conservative treatment with accelerated radiotherapy and concomitant cisplatin. Int J Cancer 2001; 96: 350-55.

12. Hussain SA, Stocken DD, Peake DR, Glaholm JG, Zarkar A, Wallace DM, James ND. Long-term results of a phase II study of synchronous chemoradiotherapy in advanced muscle invasive bladder cancer. $\mathrm{Br} J$ Cancer 2004; 90: 2106-11.

13. Trotti A, Colevas AD, Setser A, et al. CTCAE v3.0: development of a comprehensive grading system for the adverse effects of cancer treatment. Semin Radiat Oncol 2003; 13: 176-81.

14. Kaplan ME, Meier P. Nonparametric estimation from incomplete observations. J Am Stat Assoc 1958; 53: 457-81.

15. Stein JP, Lieskovsky G, Cote R, et al. Radical cystectomy in the treatment of invasive bladder cancer: long term results in 1,054 patients. J Clin Oncol 2001; 19: 666-75.

16. Mehrsai A, Mansoori D, Taheri Mahmoudi M, Sina A, Seraji A, Pourmand $\mathrm{GH}$. A comparison between clinical and pathologic staging in patients with bladder cancer. J Urol 2004; 2: 22-6

17. Cheng L, Neumann RM, Weaver AL, et al. Grading and staging of bladder carcinoma in transurethral resection specimens. Correlation with 105 matched cystectomy specimens. Am J Clin Pathol 2000; 113: 275-9.

18. von der Maase H, Hansen SW, Roberts JT, et al. Gemcitabine and cisplatin versus methotrexate, vinblastine, doxorubicin, and cisplatin in advanced or metastatic bladder cancer: results of a large, randomized, multinational, multicenter, phase III study. J Clin Oncol 2000; 18: 3068-77.

19. Yavuz AA, Yavuz MN, Ozgur GK, Colak F, Ozyavuz R, Cimsitoglu E, Ilis E. Accelerated superfractionated radiotherapy with concomitant boost for invasive bladder cancer. Int J Radiat Oncol Biol Phys 2003; 56: 734-45.

20. Pos FJ, van Tienhoven G, Hulshof MC, Koedooder K, González González D. Concomitant boost radiotherapy for muscle invasive bladder cancer. Radiother Oncol 2003; 68: 75-80.

21. Pos FJ, Hart G, Schneider C, Sminia P. Radical radiotherapy for invasive bladder cancer: what dose and fractionation schedule to choose? Int J Radiat Oncol Biol Phys 2006; 64: 1168-73.

22. Moonen L, vd Voet H, de Nijs R, Horenblas S, Hart AA, Bartelink H. Mus cle-invasive bladder cancer treated with external beam radiation: influence of total dose, overall treatment time, and treatment interruption on local control. Int J Radiat Oncol Biol Phys 1998; 42: 525-30.
23. Horwich A, Dearnaley D, Huddart R, Graham J, Bessell E, Mason M Bliss J. A randomized trial of accelerated radiotherapy for localized invasive bladder cancer. Radiother Oncol 2005; 75: 34-43.

24. Weiss C, Rödel F, Ott O, Engehausen DG, Papadopoulos T, Sauer R, Rödel C. Pretreatment proliferation and local control in bladder cancer after radiotherapy with or without concurrent chemotherapy. Strahlenther Oncol 2007; 10: 552-6.

25. Hagan MP, Winter KA, Kaufman DS, et al. RTOG 97-06: Initial report of a phase I-II trial of selective bladder conservation using TURBT, twice-daily accelerated irradiation sensitized with cisplatin, and adjuvant MCV combination chemotherapy. Int J Radiat Oncol Biol Phys 2003; 57: 665-72.

26. Kaufman DS, Winter KA, Shipley WU, et al. Phase I-II RTOG study (99-06) of patients with muscle-invasive bladder cancer undergoing transurethral surgery, paclitaxel, cisplatin, and twice-daily radiotherapy followed by selective bladder preservation or radical cystectomy and adjuvant chemotherapy. Urology 2009; 73: 833-7.

\section{Address for correspondence}

\section{Jadwiga Nowak-Sadzikowska}

Centre of Oncology - Maria Skłodowska-Curie Memorial Institute

Krakow Branch

ul. Garncarska 11

31-115 Kraków, Poland

e-mail: z5sadzik@cyfronet.krakow.pl

Submitted: $\quad$ 28.08.2012

Accepted: $\quad$ 10.01.2013 